\title{
Genetic relatedness and its causal role in the evolution of insect societies
}

\author{
Tuomas K Pernu ${ }^{1,2,3 *}$ and HeikKi Helanterä ${ }^{4,5}$ \\ ${ }^{1}$ Helsinki Collegium for Advanced Studies, University of Helsinki, P.O. Box 4, 00014 University of Helsinki, \\ Helsinki, Finland \\ ${ }^{2}$ Department of Philosophy, King's College London, London, UK \\ ${ }^{3}$ Molecular and Integrative Biosciences Research Programme, Faculty of Biological and Environmental \\ Sciences, University of Helsinki, Helsinki, Finland \\ ${ }^{4}$ Organismal and Evolutionary Biology Research Programme, University of Helsinki, Helsinki, Finland \\ ${ }^{5}$ Ecology and Genetics Research Unit, University of Oulu, Oulu, Finland \\ *Corresponding author (Email, tuomas.pernu@helsinki.fi)
}

MS received 12 July 2018; accepted 16 April 2019; published online 17 August 2019

\begin{abstract}
The role of genetic relatedness in social evolution has recently come under critical attention. These arguments are here critically analyzed, both theoretically and empirically. It is argued that when the conceptual structure of the theory of natural selection is carefully taken into account, genetic relatedness can be seen to play an indispensable role in the evolution of both facultative and advanced eusociality. Although reviewing the empirical evidence concerning the evolution of eusociality reveals that relatedness does not play a role in the initial appearance of helper phenotypes, this follows simply from the fact that natural selection - of which relatedness is a necessary component - does not play a causal role in the origin of any traits. Further, separating two logically distinct elements of causal explanation - necessity and sufficiency - explains why the debate lingers on: although relatedness plays a necessary role in the evolution of helping and advanced eusociality, relatedness alone is not sufficient for their appearance. Therefore, if the relatedness variable in a given data set is held at a uniformly high value, then it indeed may turn out that other factors occupy a more prominent role. However, this does not change the fact that high relatedness functions as a necessary background condition for the evolution of advanced eusociality.
\end{abstract}

Keywords. Behavioral ecology; causal explanation; causal necessity; causal sufficiency; causation; group selection; inclusive fitness; kin selection; levels of selection; models; natural selection; necessary conditions; sociobiology

\section{Introduction}

The role of genetic relatedness in social evolution, especially in the evolution of eusociality in insects, has recently come under critical attention (Wilson and Hölldobler 2005; Wilson and Wilson 2007; Wilson 2008; Nowak et al. 2010; Allen et al. 2013; Wilson and Nowak 2014; Nowak and Allen 2015). This argumentation has also been extensively criticized (e.g., Abbott et al. 2011; Boomsma et al. 2011; Liao et al. 2015). Much of the criticism and ensuing discussion has revolved around the relative merits of different modeling approaches (Rousset and Lion 2011; Birch 2014; Birch and Okasha 2014), semantic issues surrounding cooperation and altruism (West et al. 2007; Boomsma and Gawne 2017), and most recently, the interpretation of different formulations of inclusive fitness approaches and their causal implications (Allen et al. 2013; Birch 2014; Birch and Okasha 2014).

However, one central claim of the argumentation merits further critical attention. This is the claim that the genetic relatedness of eusocial organisms has played no causal role in the evolution of eusociality. ${ }^{1}$ Here is a representative series of quotes illustrating this line of argumentation:

'[T]he known background biology of the eusocial insects, in particular the hymenopterans, gives no reason to presuppose that pedigree kinship is a key

\footnotetext{
${ }^{1}$ Note that the value of 'eusociality' as a term describing insect sociality has been heavily criticized by Boomsma and Gawne (2017), who make a strong case for more precise terminology and specification of the traits whose evolution is being analyzed. Thus, while the articles under analysis here do not necessarily make such distinctions, we separate, whenever possible, between facultative eusociality (societies with reproductive division of labor that is behavioral and at least partly reversible, and thus conceptually similar to various forms of cooperative breeding) and obligate or advanced eusociality ('organismal' societies with physically separated castes and irreversible reproductive division of labor).
} 
causative element in the origin and early evolution of eusociality.' (Wilson 2008, p. 22)

'[W]hile close pedigree kinship among group members inevitably accompanies the origin and early evolution of eusociality, the association is a by-product of preadaptation and not a causative condition.' (Wilson 2008, p. 22)

'Two conditions working together, key preadaptations and strong proportionate group selection, are from the evidence necessary and sufficient for eusociality. Close genetic relatedness and collateral kin selection are not necessary.' (Wilson 2008, p. 22)

' $[R]$ elatedness is better explained as the consequence rather than the cause of eusociality.' (Nowak et al. 2010, p. 1060$)^{2}$

'Grouping by family can hasten the spread of eusocial alleles, but it is not a causative agent.' (Nowak et al. 2010, p. 1060)

'While similarity of genomes by kinship was an inevitable consequence of group formation, kin selection was not the cause. The extreme limitations of kin selection and the phantom-like properties of inclusive fitness apply equally to humans and to eusocial insects and other animals.' (Wilson 2014, p. 74)

'Does 'relatedness' cause evolution of eusociality?'

(Nowak and Allen 2015, p. 3/5)

These statements are regrettably vague, even in their original contexts. We think, however, that by voicing them the critics intend to make novel and substantial claims about the evolution of eusociality, and that statements like these form the conceptual kernel of their argumentation. It is generally agreed, as will be explained in more detail below, that relatedness between reproducing individuals and their helpers has been high when eusociality has evolved. But it has been wrong to think, the critics seem to suggest, that this would imply that genetic relatedness is a causal factor in the evolution of eusociality. Is this really so? Has the established view in fact assumed that genetic relatedness is such a factor? And, if it has, has it made a mistake in doing so? What, in the first place, does it mean to claim that relatedness is, or is not, a causal factor in the evolution of eusociality? It seems that these are the central questions to be addressed in order for the discussion on the causal role of relatedness to make progress.

We aim to clear the question on the causal role of relatedness in the evolution of insect societies by relating the issue to a more encompassing conceptual view on the theory of evolution. We think that the key to taking steps towards

\footnotetext{
2 This claim, almost verbatim, is also made by Wilson and Hölldobler
} (2005, p. 13367) and Wilson and Wilson (2007, p. 340). resolving the issue lies in the intersection of conceptual analysis and empirical biology: we intend to not get lost in the technical details of the relative merits of different modeling approaches, but instead present a general analysis, and provide a framework with both conceptual and empirical dimensions to help us to assess the claims about the causal role of relatedness in the evolution of eusociality.

Our discussion proceeds in three steps. First, we examine in detail the role of relatedness in the evolution of eusociality in the light of a widely accepted general formulation of the theory of evolution by natural selection. We argue that there are purely conceptual reasons to think that genetic relatedness must feature as a necessary component in the evolution of eusociality. Whether this component is interpreted causally, however, depends on the general stance one takes on the causal role of natural selection in evolution.

Second, we present a widely accepted and empirically backed up scenario for the evolution of obligate eusociality and examine the role of genetic relatedness in it. It is noted that although it is true that relatedness does not play a role in the initial appearance of facultative or obligate helper phenotypes, this follows simply from the fact that natural selection - of which relatedness is a necessary component does not play a causal role in the origin of any traits. Hence, on a closer analysis disavowing the causal role of relatedness in the evolution of eusociality is either trivially false or trivially true: on the one hand, as a necessary component of natural selection, genetic relatedness must play an indispensable role in the evolution of eusociality; on the other, genetic relatedness - due to its very role as a necessary component of natural selection - cannot play a role in the initial appearance of eusociality. These results follow simply from the conceptual structure of the theory of evolution by natural selection.

Third, we analyze causal claims to consist of two logically distinct components: the necessity element and the sufficiency element (cf. Mackie 1965, 1974; Pernu and Helanterä 2019). When we say that $X$ causes $Y$, we often equivocate between two distinct claims: that removing $X$ will result in the removal of $Y$ (necessity), and that bringing about $X$ will result in the bringing about of $Y$ (sufficiency). Keeping this distinction in mind will turn out to be particularly useful in this context. Although genetic relatedness can be shown to be a necessary condition for the evolution of eusociality, it clearly is not sufficient. Thus, some of the critical claims can be interpreted to be true if causation is interpreted in latter terms. This is a rather empty point however, for it has never been maintained that relatedness alone would be sufficient for the evolution of any kind of altruistic behavior. However, many of the critical claims can now also be shown to be unequivocally false under the former interpretation.

Let us make a few terminological clarifications before moving on. Although we think that the lessons of this discussion are relevant to the topic of evolution of social behavior in general, we mainly use examples from hymenopterans because their evolutionary history is the 
most clearly articulated one in the currently existing literature. Eusociality refers to reproductive division of labor among separate individual organisms within groups. In the case of facultative eusociality, this division of labor is behavioral and reversible, whereas in advanced, obligate eusociality the reproductive division of labor is morphological and irreversible, so that the reproductive and nonreproductive castes are permanently committed to their roles, and the society does not function without both castes being present. Altruism is a more general notion that refers to a type of behavior displayed by an individual organism, namely behavior that benefits other organism(s) at the cost of the altruistic organism. It is clear that there is a wide range of social behavior in the animal kingdom (e.g., cooperation, reciprocity, mutualism, synergism) that differ from the genuinely altruistic behavior because they accrue direct benefits to the cooperating individuals (West et al. 2007). Whether these sorts of behavior are really altruistic or not is not of concern in here.

\section{Relatedness and natural selection}

Let us start from a common ground. Although the issue is not explicitly addressed in the debate, it is clear that all parties in it presuppose that the traits underlying facultative (helping behavior) and obligate eusociality (physically differentiated castes) are adaptations. That is, eusocial syndromes have evolved by means of natural selection. In other words, eusociality is not a 'spandrel' (sensu Gould and Lewontin 1979).

There should also be no disagreement with respect to what evolution by natural selection fundamentally amounts to. Let us start with a textbook definition of natural selection: natural selection is 'any consistent difference in fitness among phenotypically different classes of biological entities' (Futuyma 2005 , p. 251). Fitness, in turn, is the average number of offspring, or realized reproductive success of any given type of biological entity. When social traits are being considered (inclusive) fitness includes both a direct component (own offspring produced without the help of others) and an indirect component (offspring of kin that is reared through the efforts of the focal individual). Fitness values can be attributed to particular types of organisms as their mean reproductive success: an individual organism of a particular type has a certain probability of producing a given amount of own or collateral offspring, or an expected (inclusive) fitness value.

What should also be clear is that natural selection may well operate in the absence of evolution. This is again a basic truism, but one that touches the core of the current debate. Suppose we have variation in fitness in certain types of biological entities. What this means is that certain types of entities - organisms with a particular trait - are reproductively more successful than some other types of entities. What this does not mean, however, is that the first type of entities would increase their relative number in the given population. Why? Simply because it was not assumed that the offspring would be of the same type as their parents. I.e., it was not assumed that the trait in question is heritable.

What we are arriving at are the three basic components of evolution by natural selection. A population $P$ is evolving by natural selection with respect to a trait $T$ if and only if:

1. There is variation in $P$ with respect to $T$.

2. Variation in $T$ is associated with variation in fitness in $P$.

3. Variation in $T$ is heritable in $P$.

For evolution by natural selection to occur, all of these are necessary and none of them alone is sufficient. This is one of the most fundamental ideas of evolutionary theory, and as such should not be under dispute. Since those doubting the causal role of relatedness in the evolution of eusociality have explicitly stressed that they base their arguments primarily on population genetics (e.g., Nowak et al. 2011), we take it that they must be subscribing to this basic idea as well.

However, now problems arise. By simply substituting (facultative or advanced) 'eusociality' for $T$ we get the following. In order for eusociality to have been evolved by natural selection, there must have been variation with respect to traits that comprise eusociality, this variation must have had fitness consequences - eusocial organisms must have been reproductively more successful than other types of organisms - and these traits, and the variation in them, must have been transmitted to future generations. Once these elements are in place, eusocial behavior will become prevalent (in the given population). But all of these are needed; none of them is superfluous. In particular, unless the traits were heritable, they would not have spread. As already stated, the defining feature of eusociality is the reproductive division of labor where only a few organisms reproduce by the help of sterile altruists. So the question springs up: how could evolution by natural selection have brought about and maintained such altruism? If organisms with a trait $T$ do not produce offspring bearing the same trait, then it is clear that such a trait cannot become prevalent by means of natural selection. Since helpers are not reproducing, it seems conceptually impossible that such a behavior would have been evolved by natural selection.

Such a conclusion would go against the original assumption: it has been taken for granted, by all parties, that eusociality is an adaptation. The only way how that assumption could be true, it now seems, is that the genes underlying behavior are transmitted to future generations by a route other than direct reproduction. But how could that happen? Here is where the ways part. The conventional reply would invoke the notion of inclusive fitness (and relatedness between the parties of the helping behavior as a key component of inclusive fitness): although the helpers are not reproducing themselves, by helping their close relatives (who carry genes identical by descent, but which remain unexpressed in them) to breed they are indirectly passing on their own genes to the next generation. Since there is a high probability that the close relatives that are receiving the help 
of the altruists share the same genes as the altruists, the altruistic behavior passes on to the next generation through the offspring of the close relatives. In this way, a behavior that seems maladaptive when only direct fitness is considered can evolve and be maintained by natural selection: it increases the reproductive success of an individual, and the genetic similarity between the parties of the helping behavior ensures the recurrence, or inheritance, of the trait in the future generations (as discussed in detail in Section 3.3).

However, the critics now deny this explanation. According to them close genetic relatedness has not played a causal role in the evolution of eusociality. Instead of invoking inclusive fitness, it is claimed that individual selection on the genotype of the queen, and later, as eusociality has become obligate through differentiated castes, selection on the level of nests, is all that is needed for eusocial behavior to evolve. What they do not deny is that we can now observe high degree of relatedness among group members in eusocial populations. That is again something that is not under dispute. It's just that according to the critics this high degree of relatedness that we now observe is a consequence, rather than a cause, of the evolution of eusociality. This, however, seems like a very baffling claim to make in the light of what the theory of natural selection amounts to.

Now, to be clear, the statements made by the critics seem to lend themselves to a variety of interpretations. First, one could interpret them in the light of a more general claim according to which natural selection is not a causal factor in evolution. Hence, relatedness would fail to be causally related to the evolution of eusociality simply because natural selection fails to be causally related to the evolution of any traits (and relatedness is a necessary part of natural selection). We think that this claim is in fact at the conceptual heart of the matter, and we will discuss it below (this section). Second, one could try to interpret the statements as amounting to granting a causal role to natural selection, but holding on to an idea that heritability - and hence relatedness - is not a causal component in natural selection. However, it is difficult to make sense of this claim: heritability is a necessary element of evolution by natural selection, not something that is additional or supplementary to it, and hence granting a causal role to natural selection while denying such a role from one of its legs does not seem to amount to a coherent position. Third, one could interpret the statements to claim that even though both natural selection and heritability as its necessary component are causal factors in the evolution of eusociality, it is wrong to think that genetic relatedness is necessary for heritability. We take this to be an interesting claim, and forming the empirical heart of the matter. It is true that what the theory of evolution by natural selection requires is not, strictly speaking, that all the relevant information is passed on from generation to generation genetically - Darwin (1959) himself did not have any knowledge of genes; the basic idea of the theory is more abstract. It is therefore certainly not incoherent to maintain that there could be inheritance without genetic relatedness. However, in this particular case, claiming that there would be inheritance without genetic relatedness is lacking adequate empirical backing, as discussed in more detail below (Section 3.3.2). Fourth, one could take the statements to claim that relatedness is insufficient for eusociality to evolve (Liao et al. 2015). Given that it is widely recognized that there are numerous organisms that live in high relatedness (even clonal) groups, but are not eusocial, relatedness is clearly not sufficient for eusociality to evolve. Other biological factors must therefore also play a role in the process. But none of this should come as news to anybody, since ecological factors have been assumed to play an essential part in social evolution theory since Hamilton's seminal papers (Hamilton 1964). Moreover, noting that relatedness is not sufficient for eusociality to evolve only highlights the fact that it seems to be necessary, and that is the idea that the critics should be attacking. Nevertheless, understanding that appealing to necessity and sufficiency constitutes distinct causal claims holds the key to unraveling the debate, as shown in Section 4.

Supposing that these interpretations exhaust all the available options, there seems to be two significant claims to address. The first concerns the conceptual issue of the causal status of natural selection in evolution. The second concerns the empirical issue of genetic relatedness as a component of heritability. Supposing that heritability of a trait is a necessary part of any evolutionary explanation that invokes the notion of natural selection, the critics owe us an account of how eusociality is heritable without close intracolonial relatedness. Since 'heritability' and 'genetic relatedness' are not synonymous, as already noted, in principle one could accept all the three fundamental conditions of evolution by natural selection, but deny that genetic relatedness plays a role in the evolution of eusociality. However this would mean that the necessary correlation between parent and offspring would have to rise through non-genetic means. We discuss in the next section why such an 'extended inheritance' scenario is unlikely (Section 3.3.2). In the remainder of this section we will highlight the theoretically problematic issues the critics of the conventional explanation are facing.

The fundamental conceptual question is this: if a trait must be heritable in order for it to have been evolved by means of natural selection, is there any sense in claiming - or denying - that the heritability of the trait is causing its evolution? We think not. Such claims involve a conceptual misunderstanding about the structure of the theory of evolution.

The fundamental conditions of evolution by natural selection are definitional elements of the notion. That is, evolution by natural selection is not something that follows from the conditions, or something that is caused by them. Rather, evolution by natural selection is those conditions. Whenever all the three conditions hold, evolution by natural selection is in operation, but not as something that comes after, or over and above, the three conditions it is composed out of. Hence heritability, being one of those conditions, is 
never causing the evolution of any traits, not because it has no role in evolution, but because its function in the process is wholly conceptual.

What also follows from this, we think, is that evolution by natural selection is a wholly statistical process. Given a population where the fundamental conditions of evolution by natural selection hold, the population evolves by necessity (barring counterbalancing effects of mutation, drift or migration). Neither the components of evolution by natural selection nor natural selection itself are causes of this evolution. Evolution by natural selection is simply a statistical process that the given population undergoes when the fundamental conditions happen to hold.

Although all this seems to follow from the notion of natural selection rather trivially, we acknowledge that it may come to clash with some well-established intuitions with respect to evolution by natural selection - namely the idea that it is exactly natural selection that is the primary cause, mechanism or force of evolution (cf. Mayr 1982; Sober 1984; Futuyma 2005). If evolution by natural selection is a process by which evolution happens, then, strictly speaking, it does not seem to function as its cause. Whether natural selection is a cause of evolution has been heavily debated (Walsh et al. 2002; Reisman and Forber 2005). We will not indulge in this debate here. We only note that although there might be a sense in which natural selection and evolution are causally related, the fundamental statistical nature of evolutionary theory should not be questioned, and moreover: even if you would prefer the causal interpretation over the statistical, that would only force you to conclude that genetic relatedness is causally related to the evolution of altruistic traits of social insects (as a necessary component of evolution by natural selection).

There is also another debate that relates directly to the issue at hand. This is the debate about the positive vs negative role of natural selection in evolution. Although it might seem appealing to think that natural selection gives an explanation for the phenotypic traits of organisms (Neander 1995), its role should actually be seen wholly negatively (Cummins 1975; Sober 1984, 1995; Dretske 1990). That is, natural selection does not act by creating adaptive traits, but only by eliminating those that are not adaptive. This is again something that seems to follow directly from the fundamentally statistical nature of evolutionary theory. But then it is again rather obvious that relatedness does not play a causal role in the evolution of helping behavior or worker castes: natural selection is simply not contributing positively to the origin of any traits.

Therefore, there are at least two conceptual issues that those in doubt of the causal role of relatedness in the evolution of altruistic traits in social insects must face. First, heritability and in this context genetic relatedness - is a necessary condition of evolution by natural selection. As such, genetic relatedness has to play an indispensable role in the evolution of altruistic traits in social insects. Second, given the fundamentally statistical nature of the theory of evolution, it does not seem to make sense to talk about the causal role of heritability or natural selection. Hence, denying the causal role of relatedness does not seem to amount to a substantial claim. The challenge the critics are facing could therefore be presented as a dilemma: either evolution by natural selection is understood as a causal process, in which case genetic relatedness has to be construed as a part of this causal process in the evolution of altruistic traits in social insects, or evolution by natural selection is a wholly statistical process, in which case genetic relatedness should not be understood to be causally related to the evolution of altruistic traits in social insects simply because evolution by natural selection is not a causal process at all.

\section{Evolutionary scenario for advanced eusociality}

In the following, we review the role of relatedness, and address the question whether it can be interpreted in causal terms, at the key stages of evolution of advanced eusociality, using Hymenoptera as an example. We present an evolutionary scenario from a solitary ancestor, through facultative helping to a species with advanced eusociality (or superorganismality) with morphologically separated queen and worker castes, where workers have lost the option of independent reproduction (Wheeler 1911; Boomsma and Gawne 2017). This scenario is similar to the ones recently proposed by Nowak et al. (2010) and Hunt (2011). It is widely agreed that the ancestors of advanced eusocial hymenopterans were solitary wasps and bees with a nest and exhibiting maternal care of offspring. This is the subsocial route to eusociality, i.e., associations of mothers and helper daughters. The alternative parasocial, or semisocial route (where the same generation females associate with breed cooperatively), has in the light of empirical evidence never led to advanced eusocial species (Bourke 2011).

To facilitate the dissecting of the role of relatedness in evolution of advanced eusociality, we have split the evolutionary scenario into four stages (closely following Nowak et al. 2010; Bourke 2011; Hunt 2011; Wilson and Nowak 2014). Our aim is to make it clear that even if relatedness does not play a role in all of the steps - and is clearly not alone sufficient for the evolution of eusociality - it plays an indispensable role at a crucial stage, and is thus a necessary condition for the evolution of eusociality.

\subsection{First stage: Group formation and preadaptations}

As a first stage in the evolution of advanced eusociality, we need to consider a suite of traits present in the ancestral state before the helper phenotype arises. These include a defensible nest, overlapping generations, parental care by the mother, and the presence of siblings in need of help when offspring emerge. Phylogenetic reconstructions also suggest that the ancestors of each independent origin of advanced eusocial 
Hymenoptera had monogamous mothers, which guarantees high relatedness within the group (Boomsma et al. 2011 and references therein). It is clear that relatedness plays no causal role in the emergence of these preadaptive traits. However, relatedness is an inseparable part of the family structure of the mother-offspring associations.

The preadaptations can be ordered on a scale of salience with respect to the evolution of helpers (Hunt 1999, 2011). Single mating that guarantees maximally high relatedness to siblings for the helpers has been argued to be a trait of low salience, since it may well have been shared by many lineages that did not evolve sociality (Nowak et al. 2010; Hunt 2011). However, low salience, the possible lack of statistical association between eusociality and ancestral monogamy, and the fact that high relatedness per se has not been the reason why monogamy occurs in these taxa (monogamy can be selected for due to, e.g., costs of mating, as outlined in Wilson and Nowak 2014) does not change the necessary role of relatedness in subsequent stages of the evolution of eusociality, as outlined below. Furthermore, as pointed out by Boomsma and Gawne (2017), even if single mating is widely shared among lineages that did not evolve eusociality, maximal relatedness $(0.75$, i.e., full-siblings) for the whole lifetime of the helpers, so that their singly mated mother is the sole reproductive one for the group lifetime, seems to be limited to the groups where advanced eusociality has evolved.

\subsection{Second stage: Emergence of a facultative helper phenotype}

A scenario for the emergence of a facultative helper phenotype that seems likely in the face of current genetic evidence (Hunt and Amdam 2007; Toth et al. 2007) is the cooption of maternal care behavior to allomaternal care, through, e.g., modifications of dispersal and diapause behaviors (Hunt 2011). This fits the view that helper phenotypes are facultatively expressed with no fixed genetic differences among castes (an allele for obligate altruism would not spread in the population; Queller and Strassmann 1998). Lack of genetic caste determination seems to be the case throughout social hymenopterans, apart from a few exceptions found in highly derived lineages (Schwander et al. 2010).

It is clear that relatedness does not play a causal role in the emergence of the helper phenotypes, and is not a phenotypic trait of the individuals or group that is interacting with the environment and causing differences in reproductive success. However, this results simply as a trivial consequence of the evolutionary theory. Evolution by natural selection, and relatedness as its element, does not play a causal role in the origin of any phenotypic traits. According to the received view (of the modern synthesis) new traits are produced through mutation or recombination. Relatedness is not causally linked to these phenomena. Whether the received view is fully able to account for the appearance of evolutionary novelties is not of concern right now ( $c f$. Pigliucci and Müller 2010). It may be that relying on mere mutation and recombination is a too pruned view of the evolution of novelties. However, what is missing from the picture, if anything, is the ontogenetic point of view. Relatedness clearly is not a part of developmental processes either. Thus, it can be safely assumed that even if an 'extended synthesis' is called for to account for evolutionary novelties, relatedness does not come to play a causal role in the initial appearance of eusociality.

\subsection{Third stage: Spread of the helper phenotype in the population; entrenchment of the helper phenotype in development}

In this step we consider two simultaneously occurring processes: the helper phenotype has to spread in the population, and its development has to be made robust, or 'entrenched', or 'canalised' (West-Eberhard 2003). For both of these processes, we have to consider two crucial concerns in the evolutionary process: (a) the selective benefit, or the fitness effect of a trait on the one hand and (b) its inheritance on the other.

3.3.1 Selective benefit: The helper individuals may have accrued direct fitness benefits in the early stages of eusocial evolution, e.g., by laying some of their own eggs, or by later inheriting the nest and the position as the queen. However, now it is only relevant to consider the fitness effects of investing into the individually costly helping trait, since that is the altruistic trait that we are aiming to explain (as opposed to fundamentally mutualistic or reciprocal traits). The likely routes to the benefits of helping, and the ecological scenarios under which they should be relevant, have been considered at length elsewhere (Queller 1989, 1994; Gadagkar 1990). The key feature of these scenarios is the indirect fitness benefit that the helper gains from helping her mother. Here the role of relatedness can be seen as determining the optimal behavior of the helper in terms of inclusive fitness: helping is not selected for unless relatedness between the helper and the receiver is large enough to compensate for the direct fitness losses of the helper (Hamilton 1964). Monogamy of the mother guarantees maximum relatedness between the helper and the receiver. Moreover, Liao et al. (2015) have demonstrated the necessary role of high relatedness by varying the relatedness variable in the models presented by Nowak et al. (2010) (which they claimed to show that relatedness is not an important factor in the evolution of eusociality). However, as the above discussion has tried to make it clear, it does not make much sense to couch this relationship in causal terms.

3.3.2 Inheritance: As already emphasized, the necessary role of relatedness becomes clear when the focus shifts to the heredity of traits. Unless a given trait is inherited, it will not spread through natural selection. In this particular case this 
means that if a trait of the helper increases the reproductive success of the breeding individual, it does not spread if the helper and the reproducer are not relatives (for otherwise the reproductive individual has merely a random chance to pass the gene to the next generation).

This simple genetic inheritance scenario gets more complicated if the trait is not based on simple genetic inheritance at the individual level. Under an extended view of heredity (Helanterä and Uller 2010; Danchin et al. 2011), the helper phenotype that in the standard view is based on the plastic expression of the genes carried by both the reproducer and the helper, can, for example, be induced by an environmental (abiotic, biotic or social) feature, be inherited epigenetically, or be based on coercion by the reproducer even in the absence of genetic similarity. Whether helping behavior is determined by maternal or helper genes affect the conditions under which helping behavior is selected for (Liao et al. 2015). That is, if the genes affecting helping behavior reside in the breeder - i.e., helping is controlled, or enforced by the breeding individual - even unrelated helpers may be favored by selection. This can be conceptualized as group level heritability of helping brought about by coercion. Effectively, these mechanisms can be seen as creating heritability at the level of the group phenotype and the division of labor into reproducer and helper. In other words, any mechanism ensuring a helper is present in the new group founded by a dispersing reproductive female, even if this plastic helping phenotype is not coded in the genome of the foundress, is enough to ensure high heritability of the group phenotype. Group heritability is a difficult and underanalysed issue (Okasha 2006), and even group selection treatments of social evolution may rely on heritability that is causally attributed to the individual level (Marshall 2015, p. 92). More importantly for the current analysis, there are empirical reasons why the nongenetic mechanisms that could cause group heritability are unlikely to underlie the evolution of sophisticated eusocial adaptations. This is because at the early stages of facultative eusociality, the helpers have independent reproductive options available (Hunt 2011). If the helping behavior is induced non-genetically, and is not directed at relatives, it can be predicted to be evolutionarily unstable. This is because at direct selection on the helper individual should favor genetically inherited traits that either enhance competition for reproductive position or increase the chances of opting for solitary reproduction and abandoning the group. This would result in intra-organismal conflict over the helping trait, and consequently helping behavior should be an evolutionarily transient phenomenon, and not further the evolution of eusociality. Similar logic applies to scenarios where eusociality is based on green beard cooperation (Helanterä and Bargum 2007; Bourke 2011; Queller 2011) - selection for efficient helping that results in the evolution of advanced eusociality is likely only when all of the helper genome (or its extended inheritance equivalent) has a shared interest in helping.

\subsection{Fourth stage: Transition into advanced eusociality and its elaboration}

The further the eusocial evolution proceeds, the roles of reproductive individuals and helpers diverge, and the less beneficial are the independent reproductive options available for the helpers compared to the indirect fitness benefits of helping. This is because task specialization simultaneously increases benefits of helping and decreases the expected success in outside reproduction. It follows from the inclusive fitness theory that this change in the benefit-cost ratio relaxes the requirement for constantly high relatedness within groups. At the same time, as societies grow in complexity, the developmental determination of helper phenotypes can take place increasingly earlier (Wheeler 1986; Bourke 1999; Helanterä and Uller 2019). In advanced eusocial organisms the workers have given up their independent options already during their development into adults. At this point the control of caste fate is largely in the hands of the individuals that rear the brood (Ratnieks and Helanterä 2009), who maximize their inclusive fitness through efficient colony functioning. Eusociality has evolved into a stage where it is difficult to imagine a reversal to a solitary breeding strategy. Loss of worker castes has secondarily occurred in socially parasitic 'inquiline' ants, but they are highly specialized exploiters of worker force of other social insects (Buschinger 2009).

Advanced eusocial societies are indeed very diverse in their relatedness structures (Helanterä 2016), and relatedness is not consistently high. However, comparative analyses show this diversification has happened only after workers have lost their reproductive totipotency (Hughes et al. 2008), so that low relatedness does not lead to reversal of sociality. However, even after the diversification, and the point of no return of eusocial evolution, relatedness continues to play an important role in social evolution. Relatedness among group members plays a crucial role in the dynamics of co-evolving traits in the groups, e.g., when the genotypes of other group members are a part of the environment that determines the development of brood into workers (Johnson and Linksvayer 2010; Linksvayer et al. 2012). More specifically, relatedness affects, e.g., how genes expressed only in workers, or genes with indirect phenotypic effects, respond to selection (Linksvayer and Wade 2009; McGlothlin et al. 2010; Bijma 2011). Thus, relatedness affects elaboration of social traits even when selection can effectively be seen as functioning at the group level, and societies approach 'superorganismality' (Gardner and Grafen 2009; Bourke 2011; Helanterä 2016; Boomsma and Gawne 2017). Furthermore, relatedness plays a role in affecting optimal allocation decisions in conflict contexts. That is, kin structure of the colonies crucially affects evolution of conflict traits, such as sex and caste 
allocation, and the amount of worker reproduction (Ratnieks et al. 2006). Ultimately, expression of conflicts could even affect stability of the societies - it seems possible that societies where relatedness is very low are evolutionary dead ends (Queller and Strassmann 1998; Helanterä et al. 2009), although reasons other than conflict are much more likely contributors to this in recently described cases (Lester and Gruber 2016).

\section{Necessity, sufficiency and causal claims in evolutionary context}

Reality is multifactorial. The biological realm in particular, as it is widely acknowledged, is notoriously complicated. Different pathways can typically lead to one and the same outcome, and we can always point to multiple factors that have a relevant role to play in the particular result we happen to be interested in. And which of these factors we choose to pinpoint as 'causes' depends often crucially on the more general framing of the issue at hand.

The preceding analysis has revealed that there are both conceptual and empirical reasons to conclude that relatedness functions as a necessary condition for the evolution of both facultative and advanced eusociality. Should this now lead one to claim that relatedness causes the evolution of eusociality? Or should one perhaps draw the opposite conclusion? In abstract, both of these conclusions can actually be argued to have support. The reason for this is simply that necessary conditions can sometimes be deemed causes, and other times not, depending on how the issue has been framed. And this ambivalence, it now becomes apparent, is the source of the current disagreements on the

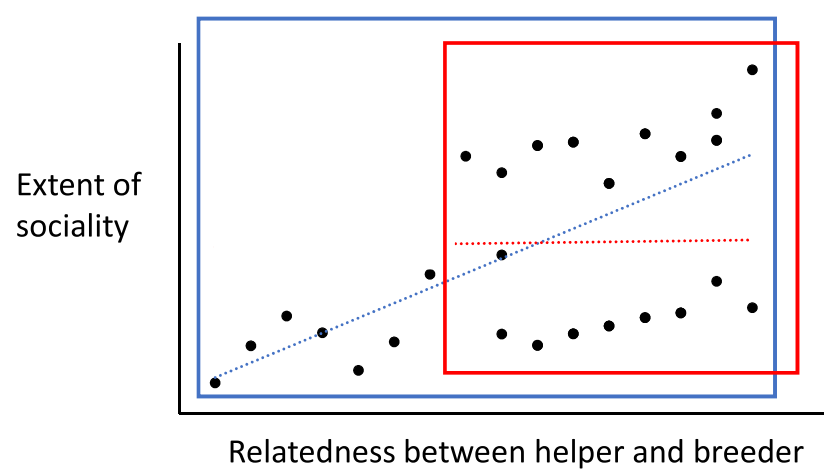

Figure 1. A hypothetical example of how the context where a question is framed affects causal conclusions, when relatedness and social behavior are continuous variables. Data in red rectangle (red line for correlation) support the hypothesis that relatedness is not sufficient for social behavior to occur, but one cannot tell, based on this information alone, whether relatedness is necessary for it to occur (as relatedness is uniformly high). Data in blue rectangle (blue line for correlation) support a necessary, but not sufficient role for relatedness, and shows a correlation with a possible causal interpretation. role of pedigree relatedness in the evolution of eusociality; the question '[d]oes 'relatedness' cause evolution of eusociality?' (Nowak and Allen 2015, p. 3/5) is in fact equivocal.

To illustrate this, consider figures 1 and $2 \mathrm{~A}$ and $2 \mathrm{~B}$. Figure 1 represents a hypothetical example of relatedness and social behavior as continuous variables. The figure shows two ways of delineating the data. The smaller, red area covers cases where relatedness is high but sociality varies. Such a data set does not contain enough information - or the relevant type of information - to draw conclusions with respect to the causal relationship between the two variables. Specifically, based on such limited information you cannot even determine whether relatedness is necessary for sociality. The data does not simply contain the relevant sort of variation to serve as a basis for answering such a question. However, the larger, blue area does contain one interesting contrast: the one between the level of relatedness and the level of social behavior. What such a data set would now clearly suggest is that high relatedness is necessary for social behavior: only in cases of high relatedness do we also encounter high levels of social behavior. But to interpret that dependency in causal terms we would need to supplement the data with further, more encompassing information.

Figure 2A gives a schematic representation of the phylogenetic relationship of several taxa of interest, based on the evolutionary scenario discussed in the previous section. This figure represents a highly simplified version of the actual situation we are facing in the current debate, and gives a schematic presentation of the sort of data that contains enough variation for us to reach some tangible conclusions. Figure 2B presents concrete, empirical data (outside of social insects) that fits into this schema. What we are now interested in is the relationship of three variables: relatedness, eusociality and an indeterminate variable $X$ representing a factor with some potential influence on eusociality. For simplicity, each of these variables are treated as binary, with ' + ' representing the presence of the given feature, and ' - ' its absence. What figures $2 \mathrm{~A}$ and $2 \mathrm{~B}$ suggest is that there is some significant connection between both relatedness and eusociality, and $X$ and eusociality.

The debate on the role of relatedness in the evolution of eusociality boils now down to the following question: is the value of the eusociality variable dependent on the value of the relatedness variable, or on the value of the $X$ variable? A moment's inspection should make it clear that eusociality is actually dependent on both relatedness and $X$, but with an important qualification: the presence of both relatedness and $X$ is necessary for the presence of eusociality, but only together they are sufficient. Importantly, relatedness alone is not sufficient for eusociality (the solid red taxa in figure 2A), but only adding $X$ makes it appear. And it is exactly this observation, it now seems, in which the idea that relatedness has no causal role to play in the evolution of eusociality is rooted. Inspecting figure 2A helps us to decipher what's right and what's wrong with this idea. 
(A)

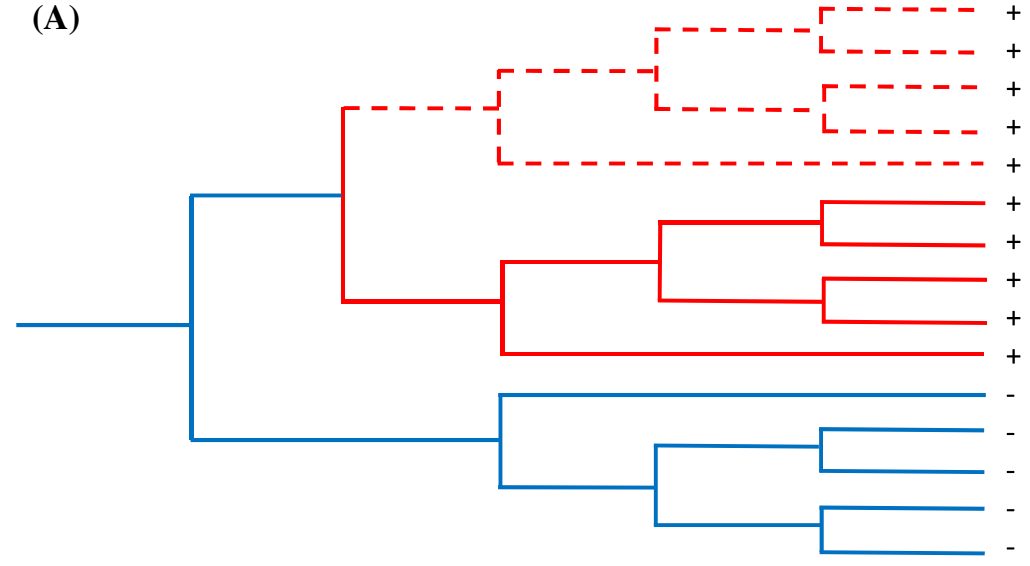

Single mating

$X \quad$ Eusocial
+

$+\quad+$

$+\quad+$

(B)

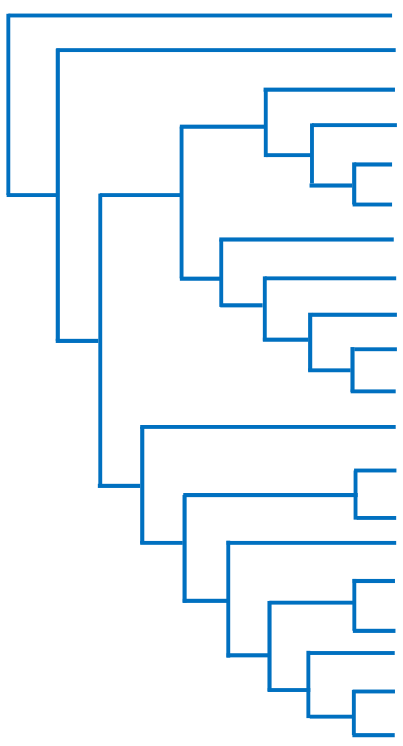

\begin{tabular}{llll} 
Species & Non-dispersal & $X$ & Eusociality \\
\hline belizensis & - & + & - \\
androsi & - & + & - \\
pectiniger & - & + & - \\
elizabethae & + & + & + \\
regalis & + & + & + \\
filidigitus & + & + & + \\
goodei & - & + & - \\
longicarpus & - & + & - \\
yano & - & + & - \\
pandionis & - & + & - \\
dardeaui & - & + & - \\
agelas & - & + & - \\
sanctithomae & - & + & - \\
williamsi & - & + & - \\
brevifrons & $\mathrm{M}$ & + & - \\
chacei & + & + & + \\
ruetzleri & $\mathrm{M}$ & + & - \\
carpenteri & $\mathrm{M}$ & + & - \\
brooksi & + & + & + \\
idios & + & + & + -
\end{tabular}

Figure 2. (A) A hypothetical example of how the context where a question is framed affects causal conclusions, when relatedness and social behavior are binary variables. Part of the phylogeny denoted by a dashed red line does not demonstrate an association with eusociality, neither for single mating nor for $X$. The part denoted by a dashed and solid red line shows an association between $X$ and eusociality, but not between single mating and eusociality. The total phylogeny shows both single mating and $X$ to be associated with eusociality, but $X$ being more salient. The full data set suggests that single mating and $X$ are both necessary, and that their co-occurrence is sufficient. In the hymenopteran context, $X$ could here be any of the traits Hunt (1999) describes as highly salient to eusociality. However, in the phylogenetic reconstructions, the absence of a sister group with low relatedness caused by multiple matings (blue lines) for comparison makes the assessment of the role of relatedness difficult (i.e., the critics base their claims on the non-existent role of relatedness on what is analogous to the red parts of the phylogeny). This is, in effect, the situation described by Hunt (1999). (B) Data from Synalpheus shrimps show that high relatedness brought by non-dispersal of larvae is necessary for eusociality to appear. However, the question whether $X$ (representing the ecological setting where eusociality has been suggested to be beneficial) too is necessary for eusociality to appear cannot be answered based on information provided by this data alone. $M=$ missing data. (Modified from Duffy and Macdonald 2009.)

Let us look at some of the concrete claims made by the critics of the kin selection theory and see how they fare in light of figure 2A. Wilson (2008) claims that '[c]lose genetic relatedness and collateral kin selection are not necessary' ( $\mathrm{p}$. 22) for the evolution of eusociality. This is unequivocally false. Many of the statements suggest either that high relatedness and eusociality are not connected at all or that eusociality is a cause of high relatedness rather than the other way around. For example, Nowak et al. (2010) claim that 'relatedness is better explained as the consequence rather than the cause of eusociality' (p. 1060). None of these claims are supported by the preceding analysis. First, there is a clear connection between relatedness and eusociality: the former is necessary for the latter. Second, nothing in figure $2 \mathrm{~A}$ 
suggests that eusociality would be the cause of high relatedness. On the contrary, the taxa represented by solid red lines suggest that the presence of high relatedness is not dependent on the presence of eusociality.

However - and this is crucial - figure 2A illustrates also clearly where some of the critical claims gain their credibility. Sometimes it is claimed that high relatedness is not a 'salient' feature, or a 'driving force' in the evolution of eusociality. For example, Wilson and Hölldobler (2005) claim that 'eusociality cannot arise without the driving force of group selection, regardless of the degree of relatedness within local populations or cooperative aggregations' (p. 13367). Nowak et al. (2010) claim that evidence has begun to accumulate that is 'unfavorable to the basic idea that relatedness is a driving force for the emergence of eusociality' (p. 1058). These claims can actually now be interpreted to be true, at least partly, in the sense that the presence of eusociality is not dependent on the presence of high relatedness. This is evident from figure 2A: there are taxa (solid red line) where relatedness is high but eusociality is absent. However, it should be noted that the claim has never been that relatedness alone would be sufficient for the evolution of eusociality: it is clear that the right sort of ecological factors will also need to be present.

Most importantly, note that if you limit your focus on the taxa where high relatedness is present (both solid and dashed red lines in figure 2A) both the presence and absence of eusociality become dependent solely on $X$ (this compares to the data set delineated by the red rectangle in figure 1). The phylogenetic reconstructions of relatedness level show that high relatedness (achieved through single mating of the mother) is the ancestral state for eusocial evolution (Hughes et al. 2008). ${ }^{3}$ As a reference group with low relatedness is typically not available (e.g., Hunt 1999), the causal role of relatedness cannot be determined by the data; high relatedness simply appears as a uniform background condition. Thus, the critics can reasonably argue that our understanding of any origin of eusociality within Hymenoptera is effectively similar to the red section of the phylogeny in figure $2 \mathrm{~A}$. In such cases it might seem natural to conclude that it is $X$ (which could be any of the more salient traits outlined by Hunt 1999), rather than relatedness, that is the cause of eusociality. But that is simply because the relatedness variable is being held constant (present). If the data does not contain variation in its value, no information is available to ground causal claims on it (neither for nor against the causal efficacy of relatedness).

Thus, for an unequivocal assessment of the causal role of relatedness, these reconstructions alone are clearly not enough. However, data from bees where social behavior is highly variable even among closely related taxa, and variation in relatedness does

\footnotetext{
3 As above, we note ( $c f$. Boomsma 2009, 2013; Boomsma and Gawne 2017) here that the analysis of Hughes et al. (2008) does not assess the role of life-time full-sibling relatedness, that seems to be limited to the advanced eusocial taxa, and thus does not account for the possibility of remating by the mother, or the possibility of the replacement of the reproductive individual, which would cause deviations from maximal life-time relatedness.
}

not derive from mating frequencies but from whether social groups are communal or mother-offspring based (subsocial), data does suggest that facultative eusocial societies have only developed from the latter, i.e., under high relatedness (Danforth 2002; Boomsma 2009). Similarly, in snapping shrimps, phylogenetic contrasts show that helping, i.e., facultative eusociality has only evolved in groups where lack of dispersal of larval individuals creates family groups where helping behavior is benefiting closely related individuals (Duffy and Macdonald 2009). Such groups, where the ecological context is invariable, but relatedness varies (figure $2 \mathrm{~B}$ ) have more power to demonstrate the necessary role of relatedness. However, these data do not contain the relevant information to assess the causal role of ecological factors in a precise manner.

Since nature is multifactorial, it makes all the difference in the world how you choose to limit your focus, and what you count in and what you leave out from your analysis. If you take all the available empirical data into account, it becomes apparent that high relatedness is necessary, but not, by itself, sufficient for eusociality. It is easy to see from figure $2 \mathrm{~A}-$ when all the information present in the figure is taken into account - that when relatedness is absent, eusociality will also be absent; the former is therefore necessary for the latter. But it is equally easy to see that when relatedness is present it is not necessarily so that eusociality will also be present; the former is therefore insufficient for the latter. However, it would be wrong to conclude from this that it is $X$ rather than high relatedness that is causally related to eusociality. According to the evidence presented in figure $2 \mathrm{~A}, X$ is causally related to eusociality only on the condition that high relatedness is present. In other words, although high relatedness by itself appears to be insufficient for eusociality, together with $X$ they form a sufficient whole: high relatedness added with $X$ brings about eusociality. But such a conclusion is totally in line with the received view on the evolution of eusociality.

\section{Conclusions}

Both theoretical and empirical considerations suggest a necessary role for relatedness in the evolution of eusociality. It is thus not clear how the seemingly provocative statements about the causal role of relatedness should be interpreted. Much depends on the more general question of how one perceives the causal status of natural selection in the process of evolution. If one subscribes to the view that natural selection is the primary causal mechanism in evolution, then one is bound to accept the view that relatedness has played a causal role in the evolution of eusociality, as a necessary element of natural selection. This view does not require that relatedness is a trait with high salience with respect to the evolution of eusociality. If, on the other hand, one sees the evolution by natural selection wholly statistically, i.e., if one disavows the causal role of natural selection in evolution, then one is bound to deny the causal role of relatedness 
in the evolution of eusociality. However, such a conclusion would follow trivially from the adopted philosophical view on the purely statistical role of natural selection in evolution, and would therefore not be very informative (at least not in the way the critics suggest).

The role of causal explanation in the theory of social evolution is an issue that has been addressed by some recent discussions (Okasha 2006; Allen et al. 2013; Birch and Okasha 2014; Pernu and Helanterä 2019), especially in terms of comparing the accuracy and adequacy of different modelling framework. Our conclusions complement these earlier discussions in important respects. Although questions on whether kin selection or multilevel selection models of social evolution are formally equivalent, and on whether the one or the other offer a more adequate causal description of the evolutionary process, are highly pertinent, our aim here has been to highlight the fact that as long as pedigree relatedness is underlying the heritability of phenotypes, in the way outlined here, giving a definite answer on questions concerning the causal role of relatedness in the evolution of eusociality depends more on one's view on causal explanation in the theory of evolution in general, rather than on the choice of modeling methods.

The fact that relatedness is a necessary condition for the evolution of eusociality does not by itself dictate a particular, unequivocal conclusion in causal terms. Whether necessary conditions are interpreted causally depends on the larger context in which such conditions are embedded. If the relatedness variable in a data set is held at a uniformly high value, then it indeed turns out that other factors become to occupy a more salient role. Since the received view on the evolution of eusociality holds only that high relatedness is necessary for the evolution of eusociality, it is perfectly consistent with this to hold that other factors will also need to be present to make eusociality actually appear. Since both high relatedness and additional factors need to be present, and they play logically distinct roles depending on different ways of delineating the relevant data, in certain situations it can actually be perfectly cogent to claim that other factors than high intracolonial relatedness appear to drive the evolution of eusociality. However, this does not change the fact that high relatedness functions as a necessary background condition for the process, and that kin selection theory is an indispensable tool for understanding the evolution of eusociality.

\section{Acknowledgements}

We would like to thank Dr Neil Bell, Prof. Kristian Donner, Dr Luke Holman, Dr Gunther Jansen, Dr Jani Raerinne, Dr Isaac Salazar Ciudad, Dr Tobias Uller and an anonymous referee of the Journal of Biosciences for helpful comments and criticism on various versions of this article. This work has been financially supported by the Academy of Finland (grants \#140990, \#135970, \#251337 and \#284666) (HH), the Ella \& Georg Ehrnrooth foundation (HH and TKP), the Emil Aaltonen Foundation (TKP), the Finnish Academy of Science and Letters
(TKP), Kone Foundation (HH and TKP) and the Waldemar von Frenckell Foundation (TKP).

\section{References}

Abbott PE, Abe J, Alcock J, Alizon S, Alpedrinha JAC, Andersson M, Andre J-B and van Baalen M 2011 Inclusive fitness theory and eusociality. Nature 471 E1-E4

Allen B, Nowak MA and Wilson EO 2013 Limitations of inclusive fitness. Proc. Natl. Acad. Sci. USA 110 20135-20139

Bijma P 2011 A general definition of the heritable variation that determines the potential of a population to respond to selection. Genetics 189 1347-1359

Birch J 2014 Hamilton's rule and its discontents. Br. J. Philos. Sci. 65 381-411

Birch J and Okasha S 2014 Kin selection and its critics. BioScience $6522-32$

Boomsma JJ 2009 Lifetime monogamy and the evolution of eusociality. Philos. Trans. R. Soc., B 364 3191-3207

Boomsma JJ 2013 Beyond promiscuity: Mate-choice commitments in social breeding. Philos. Trans. R. Soc. Lond., B, Biol. Sci. 368 20120050

Boomsma JJ and Gawne R 2017 Superorganismality and caste differentiation as points of no return: How the major evolutionary transitions were lost in translation. Biol. Rev. 93 28-54

Boomsma JJ, Beekman M, Cornwallis CK, Griffin AS, Holman L, Hughes WOH, Keller L, Oldroyd BP and Ratnieks FLW 2011 Only full-sibling families evolved eusociality. Nature 471 E4-E5

Bourke AFG 1999 Colony size, social complexity and reproductive conflict in social insects. J. Evol. Biol. 12 245-257

Bourke AFG 2011 Principles of social evolution (Oxford: Oxford University Press)

Buschinger A 2009 Social parasitism among ants: A review (Hymenoptera: Formicidae). Myrmecol. News 12 219-235

Cummins R 1975 Functional analysis. J. Philos. 72 741-765

Danchin E, Charmantier A, Champagne FA, Mesoudi A, Pujol B and Blanchet S 2011 Beyond DNA: Integrating inclusive inheritance into an extended theory of evolution. Nat. Rev. Genet. 12 475-486

Danforth BN 2002 Evolution of sociality in a primitively eusocial lineage of bees. Proc. Natl. Acad. Sci. USA 99 286-290

Darwin CR 1959 On the origin of species by means of natural selection, or the preservation of favoured races in the struggle for life (London: John Murray)

Dretske F 1990 Reply to reviewers. Philos. Phenomenol. Res. 50 819-839

Duffy JE and Macdonald KS 2009 Kin structure, ecology and the evolution of social organization in shrimp: a comparative analysis. Proc. R. Soc. B Biol. Sci. 277 575-584

Futuyma DJ 2005 Evolution (Sunderland, MA: Sinauer Associates)

Gadagkar R 1990 Evolution of eusociality - The advantage of assured fitness returns. Philos. Trans. R. Soc. London, Ser. B., Biol. Sci. 392 17-25

Gardner A and Grafen A 2009 Capturing the superorganism: A formal theory of group adaptation. J. Evol. Biol. 22 659-671

Gould SJ and Lewontin RC 1979 The spandrels of San Marco and the Panglossian paradigm: A critique of the adaptationist programme. Philos. Trans. R. Soc. London, Ser. B., Biol. Sci. 205 581-598

Hamilton WD 1964 Genetical evolution of social behaviour I. $J$. Theor. Biol. 7 1-16 
Helanterä H 2016 An organismal perspective on the evolution of insect societies. Front. Ecol. Evol. 46

Helanterä H and Bargum K 2007 Pedigree relatedness, not greenbeard genes, explains eusociality. Oikos 116 217-220

Helanterä H and Uller T 2010 The price equation and extended inheritance. Philos. Theory Biol. 2 1-17

Helanterä H and Uller T 2019 The causes of major transition: How social insects traverse Darwinian space; in Evolutionary causation: Biological and philosophical reflections (eds) T Uller and KN Laland Vienna Series in Theoretical Biology (Cambridge, MA: MIT Press)

Helanterä H, Strassmann JE, Carrillo J and Queller DC 2009 Unicolonial ants: where do they come from, what are they and where are they going? Trends Ecol. Evol. 24 341-349

Hughes WOH, Oldroyd BP, Beekman M and Ratnieks FLW 2008 Ancestral monogamy shows kin selection is key to the evolution of eusociality. Science 320 1213-1216

Hunt JH 1999 Trait mapping and salience in the evolution of eusocial Vespid wasps. Evolution 53 225-237

Hunt JH 2011 A conceptual model for the origin of worker behaviour and adaptation of eusociality. J. Evol. Biol. 25 1-19

Hunt JH and Amdam GV 2007 Bivoltinism as an antecedent to eusociality in the paper wasp genus Polistes. Science 308 264-267

Johnson BR and Linksvayer TA 2010 Deconstructing the superorganism: Social physiology, groundplans, and sociogenomics. Q. Rev. Biol. 85 57-79

Lester PJ and Gruber MAM 2016 Booms, busts and population collapses invasive ants. Biol. Invasions 18 3091-3101

Liao X, Rong S and Queller DC 2015 Relatedness, conflict, and the evolution of eusociality. PLoS Biol. 13 e1002098

Linksvayer TA, Fewell JH, Gadau J and Laubichler MD 2012 Developmental evolution in social insects: Regulatory networks from genes to societies. J. Exp. Zool. B Mol. Dev. Evol. 318 159-169

Linksvayer TA and Wade MJ 2009 Genes with social effects are expected to harbor more sequence variation within and between species. Evolution 63 1685-1696

Marshall JAR 2015 Social evolution and inclusive fitness theory: An introduction (Princeton: Princeton University Press)

Mayr E 1982 The growth of biological thought: Diversity, evolution, and inheritance (Cambridge MA: Harvard University Press)

Mackie JL 1965 Causes and conditions. Am. Philos. Q. 12 245-265

Mackie JL 1974 The cement of the universe: A study of causation (Oxford: Oxford University Press)

McGlothlin JW, Moore AJ, Wolf JB and Brodie ED 2010 Interacting phenotypes and the evolutionary process. III. Social evolution. Evolution 64 2558-2574

Neander K 1995 Pruning the tree of life. Br. J. Philos. Sci. 46 59-80

Nowak MA and Allen B 2015 Inclusive fitness theorizing invokes phenomena that are not relevant for the evolution of eusociality. PLoS Biol. 13 e1002134

Nowak MA, Tarnita CE and Wilson EO 2010 The evolution of eusociality. Nature 466 1057-1062

Nowak MA, Tarnita CE and Wilson EO 2011 Nowak et al. Reply. Nature 471 E9-E10

Okasha S 2006 Evolution and the levels of selection (Oxford: Oxford University Press)
Pernu TK and Helanterä H 2019 Social evolution and the two elements of causation. Oikos 128, 905-911

Pigliucci M and Müller GB (eds) 2010 Evolution: The extended synthesis (Cambridge MA: MIT Press)

Queller DC 1989 The evolution of eusociality: Reproductive head starts of workers. Proc. Natl. Acad. Sci. USA 86 3224-3226

Queller DC 1994 Extended parental care and the origin of eusociality. Proc. R. Soc. London, Ser. B, Biol. Sci. 259 105-111

Queller DC 2011 Expanded social fitness and Hamilton's rule for kin, kith, and kind. Proc. Natl. Acad. Sci. USA 108 10792-10799

Queller DC and Strassmann JE 1998 Kin selection and social insects. BioScience 48 165-175

Ratnieks FLW and Helanterä H 2009 The evolution of extreme altruism and inequality in insect societies. Philos. Trans. R. Soc. Lond., B, Biol. Sci. 364 3169-3179

Ratnieks FLW, Foster KR and Wenseleers T 2006 Conflict resolution in insect societies. Annu. Rev. Entomol. 51 581-608

Reisman K and Forber P 2005 Manipulation and the causes of evolution. Philos. Sci. 72 1113-1123

Rousset F, Lion S 2011 Much ado about nothing: Nowak et al.'s charge against inclusive fitness theory. J. Evol. Biol. 24 1386-1392

Schwander T, Lo N, Beekman M, Oldroyd BP and Keller L 2010 Nature versus nurture in social insect caste differentiation. Trends Ecol. Evol. 25 275-282

Sober E 1984 The nature of selection: Evolutionary theory in philosophical focus (Cambridge, MA: MIT Press)

Sober E 1995 Natural selection and distributive explanation: A reply to Neander. Br. J. Philos. Sci. 46 384-397

Toth AL, Varala K, Newman TC, Miguez FC, Hutchison SK, Willoughby DA, Simons JF, Egholm M, Hunt JH, Hudson ME and Robinson GE 2007 Wasp gene expression supports an evolutionary link between maternal behavior and eusociality. Science 318 41-44

Walsh DM, Lewens T and Ariew A 2002 The trials of life: Natural selection and random drift. Philos. Sci. $69452-473$

West SA, Griffin AS and Gardner A 2007 Social semantics: Altruism, cooperation, mutualism, strong reciprocity and group selection. J. Evol. Biol. 20 415-432

West-Eberhard MJ 2003 Developmental plasticity and evolution (Oxford: Oxford University Press)

Wheeler WM 1911 The ant-colony as an organism. J. Morphol. 22 307-325.

Wheeler DE 1986 Developmental and physiological determinants of caste in social Hymenoptera: Evolutionary implications. Am. Nat. 128 13-34

Wilson EO 2008 One giant leap: How insects achieved altruism and colonial life. BioScience 58 17-25

Wilson EO 2014 The meaning of human existence (New York: Liveright)

Wilson EO and Hölldobler B 2005 Eusociality: Origin and consequences. Proc. Natl. Acad. Sci. USA 102 13367-13371

Wilson DS and Wilson EO 2007 Rethinking the theoretical foundation of sociobiology. Q. Rev. Biol. 82 327-348

Wilson EO and Nowak MA 2014 Natural selection drives the evolution of ant life cycles. Proc. Natl. Acad. Sci. USA 111 12585-12590 